\title{
Robust experimental study of avalanche precursory events based on repro- ducible cycles of grain packing destabilizations
}

\author{
Luc Oger $^{1, *}$, Renaud Delannay ${ }^{1, * *}$, and Yves Le Gonidec ${ }^{2, * * *}$ \\ ${ }^{1}$ Univ. Rennes, CNRS, IPR [(Institut de Physique de Rennes)]-UMR 6251, F-35000 Rennes, France \\ ${ }^{2}$ Univ. Rennes, CNRS, Géosciences Rennes-UMR 6118, F-35000 Rennes, France
}

\begin{abstract}
Quasi-periodic collective displacements of grains at the free surface of a tilted grain packing constitute precursors of granular avalanches. Laboratory experiments are commonly performed by slowly tilting the packing from $0^{\circ}$ to the maximal stability angle $\theta_{A}$. In these conditions, the number of precursors is too small to assess reproducible and robust statistical analyses of the precursor activity. To go beyond this limitation, we have developed a specific experimental protocol consisting of tilting the packing with successive oscillation cycles. We use a high-resolution optical camera and process the images of the packing free surface to identify precursory events during many consecutive cycles of a single packing. We observe the same behavior for all half-cycles, forth and back: appearance of the first precursors after the same variation of inclination, exponential evolution of the weak surface activity for the first precursors and linear growth of stronger surface activity for the following ones. The experimental protocol provides both reproducible precursor measurements based on large sample statistical inferences and a quasi-stationary state after one full-cycle. This approach is very promising for highlighting the effects of external parameters, including humidity and packing geometry.
\end{abstract}

\section{Introduction}

Understanding the triggering mechanisms of granular flows is a subject of great interest in many domains. For instance, in pharmaceutical and agricultural domains, grains can be stored, pilled, or displaced; in natural domains, snow avalanches or giant landslides require prediction to prevent the recurrence of such dramatic events. At small scales, laboratory experiments are performed to study gravity destabilization of a packing of glass beads by gently inclining it, assuming a quasi-static behavior. The quasi-static regime is satisfied if the inclination process allows the non-displacement of the grains except when local shear forces reach a critical value related to the Coulomb's friction law. The destabilization is generally characterized by grain rearrangements at the free surface, based on optical measurements [1-3] but many other experimental techniques have also been successfully used (see [4] for a comprehensive presentation).

Nerone et al. [5, 6] have shown that the size of the grain rearrangements at the free surface increases with the inclination angle $\theta$ for freshly prepared packings and that quasi-periodical large collective events can be observed few degrees before the maximum stability angle associated to the avalanche $\theta_{A}$. They called such events "precursors" and observed that their size, i.e., the number of grains involved, grows exponentially with respect to $\theta$. A better understanding of the precursor activity is re-

*e-mail: luc.oger@univ-rennes1.fr

**e-mail: renaud.delannay@univ-rennes1.fr

***e-mail: yves.legonidec@univ-rennes1.fr

A video is available at https://doi.org/10.48448/wmb1-mt86 quired to predict the onset of the avalanche. The avalanche triggering and the eruption of precursors are controlled by several physical parameters, such as the packing dimensions (height [7], length [2] and width [8-11]), the density or volume compaction of the packing $[2,7,12$ $14]$, the tilting regime $[4,15,16]$ and the humidity inside the packing [17]. Most of the previous experiments $[1-3,5,7,13,17-19]$ dedicated to precursor identification have been performed by direct tilting experiments of a grain packing from the horizontal position to the avalanche.

Kiesgen de Richter et al. [20] have stated that the destabilization activity of the grain packing depends strongly on the history of the system. Information on that past history, and in particular the packing fabrication, is stored in the initial network of the physical contacts that exist between the grains. To study the influence of the system history on the precursor activity, the authors applied a series of consecutive forth-and-back tilting cycles: the box was slowly inclined from the horizontal to an "ageing" angle smaller than the avalanche angle and then inclined back to the horizontal. The authors observed a decrease of the overall precursor activity during the first cycles. After four cycles, the activity reaches a limit state that corresponds to a strongly reduced intensity. It is worth noting that, using acoustical methods, the authors observed 'mirror-type' rearrangements in the bulk of the pile. The ascending and descending parts of the cycles form a kind of anti-symmetric hysteresis loop which remains during up to 15 cycles. This behavior has motivated the present work where we implement a new experimental protocol. The 
specific protocol consists in continuously tilting the packing with successive oscillation cycles between $\theta_{C}<\theta_{A}$ and $-\theta_{C}$. As shown in the following, this original approach allows providing a large number of precursors that improves the accuracy of the analyses.

In section 2, we present the experimental setup and the techniques used to monitor the surface activity. Section 3 describes the results and presents the advantages of the successive oscillation cycles, defined as 'full-cycle', to characterize the precursor activity.

\section{Description of the reproducible cycle experiments}

\subsection{Grain packing fabrication}

To achieve the so-called "same history" of a packing fabrication [21], we have followed the approach introduced by [20] which consists in a four-steps protocol.

In the first step, we position a $1 \mathrm{~cm}$ square mesh grid at the bottom of a rectangular aluminum container with length $L_{b}=440 \mathrm{~mm}$, width $W_{b}=200 \mathrm{~mm}$ and height $H_{b}=185 \mathrm{~mm}$. Note that the dimensions of the container are adjustable $\left(L_{b}=240,440,640\right.$ or $1000 \mathrm{~mm}$ and $W_{b}=150$, 200 or $300 \mathrm{~mm}$ ). In the second step, we fill the container with monodisperse glass beads with a diameter $D=2 \mathrm{~mm}$. The number of grain layers is about 90 , which is high enough to avoid any influence on the maximal stability angle of the packing, the well-known wall effect in granular packings [22]. Note that this number is much larger that the limit of 10 layers observed by Aguirre et al. [13]. In the third step, we slowly pull out the grid, an operation which results in a nearly loose packing. In the forth step, we flatten the free granular surface of the packing by pushing horizontally a rigid metallic bar along the $L_{b}$ direction.

The average initial packing fraction (ratio between the volume of sphere material with the volume of the whole packing) evaluated in [20] is $0.594 \pm 0.001$ but the last step of the packing fabrication may compact the superficial layer of grains by only few tenths but enough to result in a "scrapped surface" of the packing.

\subsection{Experimental setup and successive tilting cycles principle}

Preliminary developments of an automatic tilting experimental setup have been designed to introduce original results in characterizing precursors and avalanches of granular piles [4, 19, 23]. For the present work, this experimental setup has been significantly improved to reduce artifacts, including mechanical vibrations and electrostatic effects between the glass beads, to consider different sizes of the packing container, to synchronize several physical measurements not discussed here, and to perform large range of inclinations positives or negatives (Fig. 1).

The experimental setup, placed in a humidity and temperature controlled room, consists of an optical table managed by four pneumatic attenuators and mounted on damping pads to reduce external vibrations that may modify the dynamics of the packing. Both the rectangular aluminum box that contains the grains, a camera used to scan the free surface and lights used to illuminate the free surface of the packing are fixed on an inclinable plate that can rotate to a maximum angle of $30^{\circ}$ around a horizontal axis made of Teflon. The box is positioned so that the horizontal axis is directed according to $W_{b}$ and is located at the center of the $L_{b}$ length of the box. The plate inclination is controlled using a linear actuator and the tilting speed can be set between 1.7 and $14^{\circ} / \mathrm{mn}$ in a LabVIEW ${ }^{\mathrm{TM}}$ program that also records the inclination angle. In the present work, the experiments have been performed at the minimum speed.

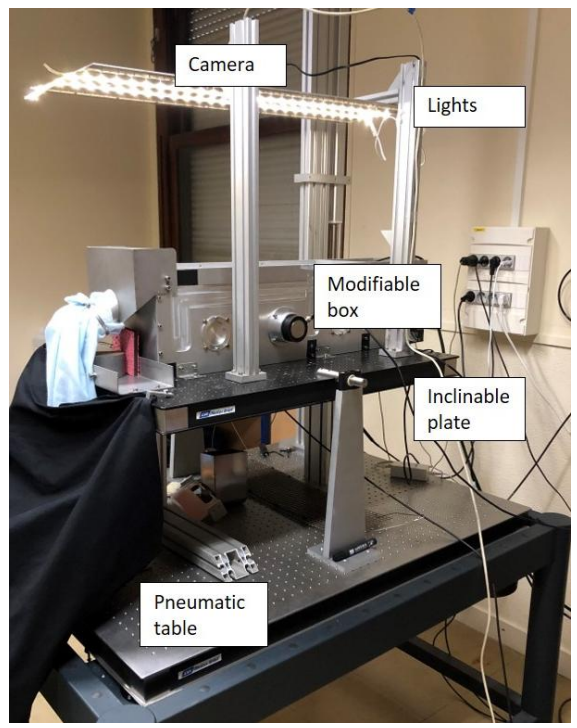

Figure 1. Photography of the experimental setup.

The experimental setup can perform multi-cycle experiments composed of an initial quarter-cycle $C_{0}$ from $0^{\circ}$ to $\theta_{C}<\theta_{A}$, corresponding to a classical tilting experiment with stop before reaching avalanche, followed by a series of successive half-cycles indexed by $i$ from $+\theta_{C}$ to $-\theta_{C}\left(C_{i}^{+}\right)$and from $-\theta_{C}$ to $+\theta_{C}\left(C_{i}^{-}\right)$. After $N$ full-cycles, the inclination of the last half-cycle $C_{i}^{-}$increases up to the maximal stability angle $\theta_{A}$.

\section{Measurement of the precursor activity}

\subsection{Optical detection of the precursory events}

During the experiment, the optical camera takes one image of the free granular surface per second with a resolution of $2448 \times 2050$ pixels on 8 bits gray level. This total surface is noted $S_{0}$. Positioned $1 \mathrm{~m}$ above the free granular surface and set on an aperture speed of $1 / 15000 \mathrm{~s}$, the camera allows imaging a surface of $230 \times 230 \mathrm{~mm}^{2}$ located at the center of the granular surface and the grain $2 \mathrm{D}$ projection corresponds to about 300 pixels. The image acquisition is performed by the LabVIEW ${ }^{\mathrm{TM}}$ program and is thus synchronized with the inclination angle measurements as a function of the recording time.

Four stripes of LEDs illuminate the packing free surface to produce grain shadows. Changes in the shadows 
surface between two consecutive images are used to detect grain displacements: an ImageJ [24] script, initially developed by Kiesgen de Richter et al. [2], consists in subtracting two consecutive images, thresholding the result to remove the noise and then quantifying the surface $S$ of the displaced grain shadows as a function of the inclination angle. Based on the ratio $S / S_{0}$ hereafter called the surface activity, the detection of a precursory event is like the approach used in previous studies [2, 5, 17, 19] and corresponds to a surface activity close to unity.

\subsection{Precursor activity in the classical range $0^{\circ}$ to $\theta_{C}$}

The initial quarter-cycle $C_{0}$ of our multi-cycle protocol is like classical avalanche experiments but the inclination is stopped before the avalanche occurs, at about $28^{\circ}$ : just after its fabrication, the grain packing is slowly tilted from the horizontal $\theta=0^{\circ}$ to $\theta_{C}=24^{\circ}$. The image processing allows detecting several main peaks of activity which appear quasi-periodically (Fig. 2, blue curve). They can thus be associated to precursory events. The red dashed line is drawn in a semi-logarithm scale to ensure the Nerone et al.'s assumption of an exponential increase [1]. Obviously, this assumption is only valid for the very first and weak amplitude precursory events. At larger inclination angles $\left(\theta>18^{\circ}\right)$, the precursor activity is getting higher but increases more slowly. It is difficult to know, without a detailed analysis, if the first small precursors, already observed by Nerone et al. [1], are of the same nature as the large ones that follow them.

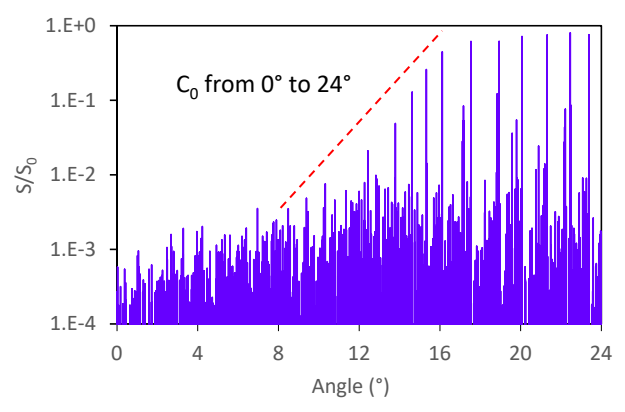

Figure 2. Surface activity measured for a $2 \mathrm{~mm}$ beads packing and $\theta_{C}=24^{\circ}$ during the initial quarter-cycle $C_{0}$. The dashed line is an exponential approximation of the activity behavior.

Weak events can also be observed between the precursory events (Fig. 2) and are discarded in the present study where we chose to identify precursory events when at least half of the free surface of the grain packing has moved. We thus characterize the dynamic of the precursor activity after the angle of the first precursor defined by $S / S_{0}>0.5$. In the present $C_{0}$ experiment which starts at $\theta=0^{\circ}$, we measure a variation $\theta_{0} \approx 16.5^{\circ}$ of the inclination angle before the first large precursor and detect $M_{0}=5$ precursors. Variations in $\theta_{0}$ and $M_{0}$ may exist from one experiment to another not only because of the use of different grain packings but also because of the noise in the image processing. When only considering $C_{0}$ experiments to characterize the precursor activity with the angle $\Delta \theta$ that separates two consecutive precursors, a large number of realizations is required to increase the total number of precursory events and get more representative statistical analyses of the precursor activity. Such an approach using different initial packings has strong limitations, in particular when we aim at quantifying the effects of external conditions (humidity, temperature, inclination speed, angle $\left.\theta_{C}, \ldots.\right)$ on the precursor activity.

\subsection{Multi-cycle precursor activity from $\theta_{C}$ to $-\theta_{C}$}

This section presents the first results of the multi-cycle experiment composed of $N=4$ full-cycles with $\theta_{C}=24^{\circ}$ (Fig. 3a).
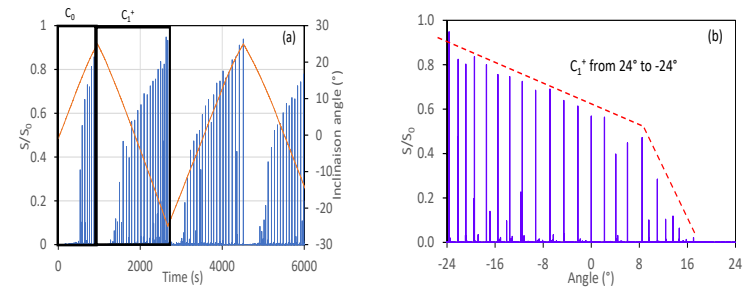

Figure 3. (a) Surface activity $S / S_{0}$ measured as a function of the recording time during a multi-cycle experiment composed of 4 full-cycles (blue line). During the first quarter-cycle and each following half-cycle, the inclination angle $\theta$ changes linearly with the time (red lines). (b) Activity of the $C_{1}^{+}$half-cycle following $C_{0}$ as a function of the inclination angle.

The experiment starts with the initial quarter-cycle $C_{0}$ discussed in the previous section, represented here as a function of the acquisition time. It is followed by the first half-cycle $C_{1}^{+}$(Fig. 3b) during which the first "large" precursor appears at $\theta \approx 8^{\circ}$. The variation of inclination angle necessary to observe this first large precursor is thus $\theta_{1}^{+}=\theta_{C}-8^{\circ} \simeq 16^{\circ}$, like $\theta_{0}$ measured in the previous case $C_{0}$. Then, we observe a long series of precursors whose activity increases up to the opposite extreme angle $-\theta_{C}$. Interestingly, the activity increases rapidly for the weak activity precursors preceding $8^{\circ}$ and then linearly at a much slower rate for the precursors of stronger surface activity, after $8^{\circ}$. This is symmetric to what we observed in Fgure 2 and confirms the mirror-type effect observed in [20]. In this first half-cycle $C_{1}^{+}$, with $\theta_{1}^{+} \simeq 16^{\circ}$, the number of precursors $M_{1}^{+}=16$ is already much larger than $M_{0}$. In the second half-cycle $C_{1}^{-}$, we observe a symmetric behavior with $\theta_{1}^{-} \simeq 19^{\circ}$, and a number of precursors $M_{1}^{-}=13$. So, in practice, we can average inter-precursor angles $\Delta \theta$ on each group of precursors during $C_{i}^{ \pm}$half-cycle or the summation of the inter-precursors angles during each half-cycle up to the given full-cycle $N$.

As a result, the total number $M_{N}=\sum_{i=1}^{N}\left(M_{i}^{+}+M_{i}^{-}\right)$ of precursory events detected for a single grain packing can be remarkably high. The results show a good reproducibility of the precursor activity after the initial quartercycle $C_{0}$ and no more than two full-cycles are required to 
get a representative grain packing behavior. Thanks to the robust statistical approach allowed by the new multi-cycle protocol, only few full-cycles are enough to obtain a good accuracy of the results. In practice, four full-cycles following the $C_{0}$ quarter-cycle will be used in our future work.
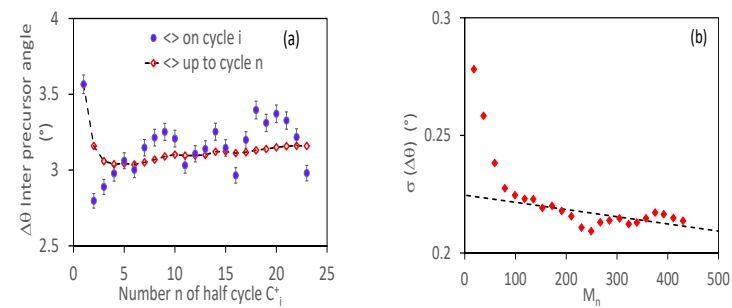

Figure 4. (a) Evolution of the inter-precursor angle $\Delta \theta$ averaged $\left(<>\right.$ )on each half-cycle $C_{i}^{ \pm}$(disk with its error bar) and accumulated average from 0 up to $n$ (open diamond) versus the number $n$ up to 23 full-cycles. (b) Evolution of the accumulated data error bar versus $M_{n}=\sum_{i=1}^{n}\left(M_{i}^{+}+M_{i}^{-}\right)$, the dashed line corresponds to the fit for the latest values.

Figure 4 demonstrates that, after the first full-cycles, we can observe a quasi-stationary of the precursor measurements for the average inter-precursor angle $\Delta \theta$ between two consecutive precursory events. We can notice also the accuracy increase with the increase of $M_{n}$ after $n$ full-cycles.

\section{Conclusion}

The original multi-cycle experimental protocol designed to study precursor activity of grain packings allows us to provide precursor measurements based on large sample statistical inferences. Additional experiments -not shown here- demonstrate that this quasi-stationary state already obtained after two full-cycles has also permitted us to demonstrate that this setup protocol doesn't seem to depend much on the initial packing fabrication. With only two full-cycles, we have highlighted this quasi-stationary state and, for accuracy and reproducibility improvement, four full-cycles will be used as standard protocol in our future experimental works. This new approach can generate accurate and reproducible results required to measure and analyze in more detail weak variations induced by changes in physical parameters such as humidity, packing geometry and inclination speed that motive future works on grain packing precursory events.

Acknowledgments We would like to thank P. Chasle for all the developments of our LabVIEWTM code and Y. Robert and G. Pecheul for the mechanical realizations.

\section{References}

[1] N. Nerone, M. Aguirre, A. Calvo, D. Bideau, I. Ippolito, Phys. Rev. E 67, 011302 (2003)

[2] S. Kiesgen de Richter, G. Le Caër, R. Delannay, J. Stat. Mech.-theory E 2012, p04013 (2012)

[3] M. Duranteau, Ph.D. thesis, Université Rennes 1 (2013)

[4] R. Delannay, M. Duranteau, V. Tournat, C. R. Phys. 16, 45 (2015)

[5] N. Nerone, M. Aguirre, A. Calvo, I. Ippolito, D. Bideau, Phys. A 283, 218 (2000)

[6] N. Nerone, S. Gabbanelli, Granular Matter 3, 117 (2001)

[7] M.A. Aguirre, N. Nerone, I. Ippolito, A. Calvo, D. Bideau, Granular Matter 3, 75 (2001)

[8] P. Boltenhagen, Eur. Phys. J. B 12, 75 (1999)

[9] S. Courrech du Pont, P. Gondret, B. Perrin, M. Rabaud, Europhys. Lett. (EPL) 61, 492 (2003)

[10] W. Bi, R. Delannay, P. Richard, N. Taberlet, A. Valance, J.Phys-Condens. Mat. 17, S2457 (2005)

[11] J. Métayer, Ph.D. thesis, Université de Rennes I (2008)

[12] P. Evesque, D. Fargeix, P. Habib, M.P. Luong, P. Porion, J. Phys. I France 2, 1271 (1992)

[13] M.A. Aguirre, N. Nerone, A. Calvo, I. Ippolito, D. Bideau, Phys. Rev. E 62, 738 (2000)

[14] N. Gravish, D.I. Goldman, Phys. Rev. E 90, 032202 (2014)

[15] A. Kabla, G. Debrégeas, J.M. di Meglio, T.J. Senden, Europhys. Lett. (EPL) 71, 932 (2005)

[16] A. Jarray, V. Magnanimo, S. Luding, Powder Technol. 341, 126 (2019)

[17] L. Oger, C. El Tannoury, R. Delannay, Y. Le Gonidec, I. Ippolito, Y.L. Roht, I. Gómez-Arriaran, Phys. Rev. E 101, 022902 (2020)

[18] V. Gibiat, E. Plaza, P. De Guibert, J. Acoust. Soc. Am. 123, 3142 (2008)

[19] M. Duranteau, V. Tournat, V. Zaitsev, R. Delannay, P. Richard, in Powders and Grains 2013 (AIP, 2013), Vol. 1542, pp. 650-653

[20] S. Kiesgen de Richter, V.Y. Zaitsev, P. Richard, R. Delannay, G. Le Caër, V. Tournat, J. Stat. Mech.theory E 2010, P11023 (2010)

[21] D. Bideau, E. Guyon, L. Oger, in Disorder and Fracture, edited by J.C. Charmet, S. Roux, E. Guyon (Springer US, Boston, MA, 1990), Vol. 204, pp. 255268, NATO ASI series (series b: physics) edn.,

[22] L. Oger, J.P. Troadec, D. Bideau, J.A. Dodds, M.J. Powell, Powder Technol. 46, 121 (1986)

[23] S. Kiesgen de Richter, Ph.D. thesis, Université de Rennes1 (2009)

[24] C. Schneider, W. Rasband, K. Eliceiri, Nat. Methods 9, 671 (2012) 